\title{
Studi sul recupero delle superfici decorate dell'architettura delle facciate della Cavallerizza e del Castello di San Giorgio in Palazzo Ducale di Mantova
}

Studies on the recovery of the decorated surfaces of the architecture of the facades of the Cavallerizza and the Castle of San Giorgio in the Ducal Palace of Mantua

\author{
Lorenzo Appolonia a , M. Chiara Ceriotti ${ }^{\text {b }}$, Daniela Lattanzi ${ }^{c}$, Antonio Mazzeri ${ }^{\text {d }}$, Barbara Scala ${ }^{\mathrm{e}}$ \\ ${ }^{a}$ Centro Conservazione e Restauro Venaria Reale, Turin, Italy, lorenzo.appolonia@ centrorestaurovenaria.it \\ ${ }^{\mathrm{b}}$ Restauratrice, ditta Consorzio ARKE', Rome, Italy, info@ consorzioarke.com \\ ${ }^{c}$ Ministero per i beni e le attività culturali e per il turismo, Segretariato regionale per la Lombardia, Milano, Italy, \\ daniela.lattanzi@beniculturali.it \\ ${ }^{\text {d }}$ Palazzo Ducale di Mantova, Mantova, Italy, antoniogiovanni.mazzeri@beniculturali.it \\ e Università degli Studi di Brescia, Brescia, Italy, barbara.scala@unibs.it
}

\begin{abstract}
The contribution aims to present the path of knowledge on the external surfaces of the Courtyard of the Cavallerizza and the Castle of San Giorgio in Palazzo Ducale in Mantua, the subject of an upcoming conservation project. In particular, the results emerged through the autopsy and stratigraphic survey of areas selected by sample, relating to the technique of execution and the constituent materials of the finishes. From this survey, the characteristics of the original structure have been defined so as to have a clear relationship with respect to integration or degradation. At the same time, following specific evidence and to support the cognitive operations carried out in situ, in-depth diagnostic investigations were carried out in order to support and clarify the contents detected previously by interpreting the scientific data on the nature of the materials and the degradation present. The re-elaboration and critical analysis of the data acquired through various in-depth techniques, as well as providing indications for intervening on the causes of degradation, has supported the designers in the development of intervention methods and in the choice of the most suitable materials for conservation, based on the state of conservation of the nearby areas and the interfaces with the substrate. The scientific data have been compared with the historiographic information in order to have an objective comparison.
\end{abstract}

Keywords: Scientific analyses, pilot construction site, conservation.

\section{Introduzione}

Il complesso museale di Palazzo Ducale di Mantova è stato individuato con decreto ministeriale 23 dicembre 2014 tra i musei nazionali dotati di autonomia, attivando un processo di riorganizzazione delle funzioni museali e della gestione degli interventi sul patrimonio edilizio.
In tale scenario si è delineato un terreno di confronto tra le istanze di valorizzazione e di conservazione che, nell'ambito delle nuove strutture museali, ha trovato modi e forme di sintesi e di reciproco rafforzamento. I fondi del programma ministeriale FSC 2014-2020 hanno permesso di affrontare in modo sistematico i temi della con- 
servazione e della valorizzazione all'interno di un processo metodologico coerente, in modo da rileggere le trasformazioni della fabbrica nel tempo, indirizzandola alla fruizione compatibile e sostenibile del complesso.

Gli interventi degli ultimi trent'anni sul palazzo non hanno seguito una posizione conservativa omogenea; questa considerazione emerge dalle osservazioni dirette e dalla lettura delle perizie dei lavori effettuati: interventi caratterizzati, di norma, da motivi d'urgenza e limitati a operazioni puntuali e parziali, con investimenti trascurabili per la parte diagnostica. Le nuove risorse economiche hanno attivato progetti di restauro e di ricerca volti sia all' applicazione di tecnologie innovative per la gestione del manufatto, sia a riconoscere e direzionare le scelte operative, alla luce della scarsa programmazione degli interventi operabile nel recente passato. Il contributo riporta i risultati di positiva collaborazione tra una fase di indagine conoscitiva e i riscontri pratici emersi dal cantiere pilota, al fine di offrire indirizzi operativi nel prossimo cantiere.

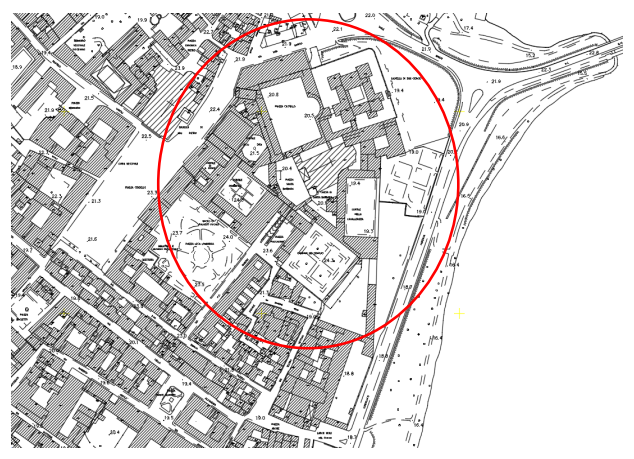

Fig. 1. Individuazione di palazzo Ducale.

\section{Il Cortile della Cavallerizza o della Mostra.}

Il Cortile della Cavallerizza era destinato alla esibizione dei cavalli dei Gonzaga. Nonostante l'apparente unità costruttiva e decorativa viene realizzato per parti successive. In una prima fase Giulio Romano costruisce i due lati corti: da un lato la loggia dei Mesi e dall'altro la Rustica. Nel 1556 risulta già realizzato il braccio di collegamento fra le due fabbriche, quello verso il lago edificato da Bertani, probabilmente su progetto di Giulio Romano. Il quarto lato la Galleria della Mostra offrirà l'occasione per innalzare tutta la struttura. È verosimile che Bertani assecondasse l'idea di realizzare un'architettura dipinta, staccandosi dal disegno di Giulio Romano come testimoniano i lacerti di finto bugnato emergenti nella loggia dei Mesi. A partire dal 1866 lo stato conservativo della Cavallerizza è documentato con maggiore precisione, grazie a Achille Patricolo curatore del palazzo. Dai carteggi emerge come il tema dell' acqua costituisse un problema importante, tanto da rendere necessario il restauro della cornice di coronamento esterna della galleria, parzialmente caduta a seguito dei danni dovuti alle infiltrazioni. Interessante è la nota che riporta come le porzioni di decorazione mancanti furono realizzate con frammenti già presenti nei depositi del palazzo, assemblati con cemento. Nonostante l'attenzione e la volontà di riordino della gestione del palazzo promossa da Patricolo, nel 1908 il Cortile della Mostra è utilizzato come campo da tennis con le conseguenti ripercussioni negative di tale uso improprio. Tra le numerose perizie presenti presso l'Archivio centrale dello Stato su Palazzo Ducale, alcune riguardano la Cavallerizza e documentano la disponibilità di fondi per i lavori di restauro. Queste perizie, seppur approvate, si attuarono solo parzialmente a causa dello scoppio della Prima Guerra Mondiale che fermò gran parte dei lavori. I tentativi di dare continuità alla manutenzione furono ostacolati dagli eventi più che dalla volontà dei responsabili.

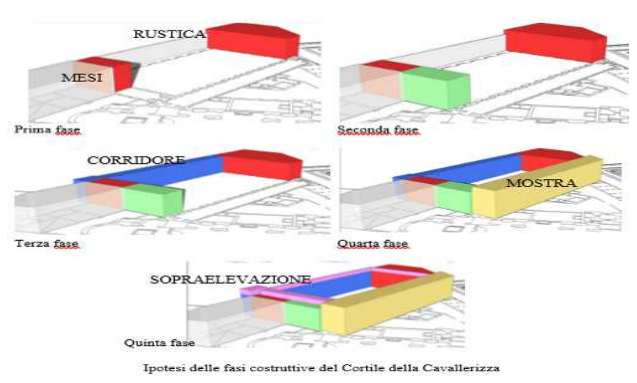

Fig. 2. Ipotesi delle fasi costruttive del Cortile della Cavallerizza.

Un utile bilancio sullo stato conservativo del Cortile della Cavallerizza lo offre Alfredo Barbacci, responsabile dal 1936 al 1939, che racconta delle condizioni conservative precarie a 
causa del suo uso ad orto. In particolare, Barbacci sottolinea come la plasticità del bugnato fosse fortemente compromessa ed i colori pressoché illeggibili.

Un primo cantiere guidato da Barbacci iniziò nel 1936 sul lato della Rustica. Di interesse sono le sue osservazioni che denotano come gli ornati plastici fossero ancora presenti ma distaccati dal supporto murario (Barbacci, 1939). La sua scelta operativa fu quella di consolidarli mediante iniezioni di "malta liquida", mentre intonaci e bugnati mancanti furono reintegrati ad imitazione degli originali, dando loro il "sapore" degli antichi. Solo per le colonne tortili Barbacci propose un approccio non imitativo: infatti i torciglioni, simulanti i tralci di vite, non furono riproposti ma la superficie venne conservata liscia. Interessante è la definizione con cui specifica la modalità operativa utilizzata, ovvero "con la tecnica antica, l'incrostazione di calce caduta o disgregata". L'intervento proseguì sul fronte della Galleria dei Mesi, in cui i basamenti delle semicolonne centrali furono ricostruiti così come i piedistalli dei davanzali delle finestre centrali. Barbacci eliminò i gradini posti in corrispondenza delle porte-finestre lungo il lato della Galleria di cui erano state già ripristinate le ringhiere in ferro. L'ultimo atto del cantiere "unitario", ovvero gestito con stessi criteri di intervento, si svolge fra il 1937 ed il 1938 e riguardano il corridore verso il lago. Le tamponature del piano terreno furono demolite e ricollocate le inferriate sulla base degli alloggi per la ferramenta nella muratura. Anche questo lato fu ripreso nelle bugne "che si rifecero con esattezza", come gli intonaci persi al primo ordine e furono integrati i restanti superiormente. Barbacci parla anche dei colori, rimasti nei toni originali all'interno delle connessure del bugnato, gialli, rossi e grigi, abbastanza smorzati. Bisogna aspettare gli anni 80 del '900 per avere nuovi interventi sulle superfici esterne, con progetti redatti dalla Soprintendenza di Brescia, competente per territorio. Purtroppo, dalle perizie conservate in archivio, non sempre si individua un'area di intervento precisa, e, quando la perizia è accompagnata da elaborati grafici, questi sono generici, rendendo incomprensibile se la procedura descritta sia stata uniformemente eseguita $\mathrm{o}$, al contrario, si sia tenuto conto delle diverse reattività dei supporti avendo la certezza della loro eterogeneità. Le descrizioni dei materiali indicati all'interno delle valutazioni di spesa sono sicuramente utili, soprattutto in vista del progetto in corso.

Una seconda fase di lavori sulle superfici iniziò nel 1994 per iniziativa del Soprintendente Ruggero Boschi, Soprintendente per i beni ambientali e architettonici per Mantova Brescia e Cremona. Dalla lettura di quest'ultimo progetto si registra una sostanziale svolta nella gestione delle opere, che asseconda le posizioni attuali. In primo luogo, è presente un documento attestante le indagini chimico fisiche atto ad individuare $\mathrm{i}$ materiali presenti, attribuendo alle tecniche di esecuzione, non del tutto congrue, e ai fattori di degrado ambientale le cause delle problematiche conservative. In particolare, si sottolinea come la granulometria dell'aggregato del rinzaffo dell'arriccio sia "eccessivamente fine per l'uso, e come gli stessi strati di preparazione siano troppo fratazzati, limitando la capacità di adesione del secondo strato d'intonaco". Il criterio di intervento proposto dalla perizia prevede puliture ad umido con nebulizzatore, pasta AB-57, o aeroabrasivi localizzati. Aspetto importante per l'attuale intervento è la conoscenza dei consolidanti utilizzati: la voce di perizia riporta: "Consolidamento generale degli intonaci tramite Primal AC-33 diluito in acqua demineralizzata [...] ricostruzione dei bugnati [...] tramite impasto di calce, inerti e pigmento, la malta come colorazione sarà ottenuta leggermente sotto tono $[\ldots]$ trattamento protettivo finale tramite idrorepellente".

\section{La fase analitica}

Ciò che viene acquisito nel percorso di conoscenza di un bene culturale è fondamentale nella fase progettuale. Le analisi di per sé possono non fornire alcun supporto e rappresentare solo il dato conoscitivo, ma, eseguite nella fase progettuale, devono rispondere a domande che riguardano le problematiche di intervento e la definizione di metodologie operative. Inoltre, la ricerca può avere come obiettivo quello di confermare o meno ipotesi di carattere storiografico. L'iter conoscitivo per l'intervento di conserva- 
zione della Cavallerizza di Palazzo Ducale di Mantova cerca di rispondere a domande riguardanti sia le fasi evolutive della fabbrica sia questioni legate al progetto di intervento. Lo studio condotto nella fase propedeutica all'intervento attraverso la realizzazione di un cantiere pilota ha consentito di entrare in contatto diretto e continuo con i materiali e le superfici che compongono gli apparati decorativi architettonici della Cavallerizza. Si premette che la riconoscibilità di quelle superfici che verosimilmente sono parti originali del progetto di Giulio Romano potrà essere esaustiva solo durante la fase di intervento vera e propria, cioè quando un ponteggio esteso permetterà una visione di insieme dei diversi lacerti che possono essere riconosciuti come giulieschi. La progettazione di questa campagna diagnostica preliminare ha richiesto un approccio applicativo, ovvero un protocollo di analisi specifico, che discende dalle domande emerse dalle fasi di mappatura del degrado. La prima domanda ha riguardato la composizione dei materiali costitutivi. L'approccio analitico nei confronti di questa tematica segue la fase di valutazione del degrado e la conseguente mappatura. Le informazioni dirette sul manufatto sono state correlate alle informazioni derivanti da studi esistenti, allo scopo di contenere il numero di prelievi da effettuare. La caratterizzazione è avvenuta in due step: una preliminare al cantiere pilota e l'altra durante il cantiere, legata alle ulteriori conoscenze e ai nuovi quesiti emersi. Questa fase ha previsto l'impiego di alcune misure invasive come il prelievo di campioni per l'analisi in laboratorio. Le analisi non invasive si sono concentrate in misure con XRF (fluorescenza di raggi $\mathrm{X}$ ) e con aquaboy, una strumentazione per la misura puntuale dell'umidità relativa di superficie. Le analisi invasive sono state condotte con l'impiego di tecniche atte alla caratterizzazione dei materiali inorganici e, in particolare, XRD (diffrazione di raggi $\mathrm{X}$ ), TG (analisi termica), IC (cromatografia ionica, adatta per la valutazione della frazione solubile come i sali) e FTIR (spettrofotometria infrarossa in trasformata di Fourier). In tabella 1 sono riportati i risultati riguardanti le analisi in XRD. Nella tabella 2 sono indcati i risultati delle analisi con cromatografia ionica.

\begin{tabular}{|c|c|c|c|c|c|c|c|c|}
\hline \multicolumn{9}{|c|}{ 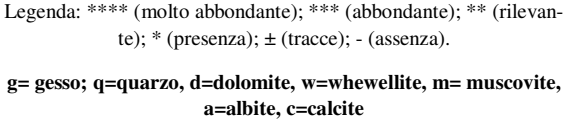 } \\
\hline & & 00 & $\sigma$ & $=$ & $\geqslant$ & $\Xi$ & $\pi$ & 0 \\
\hline As1 & $\begin{array}{l}\text { Finitura superficiale, gial- } \\
\text { lo chiara }\end{array}$ & $* *$ & $* * *$ & $* * *$ & & $*$ & $* *$ & $* * * *$ \\
\hline As2 & $\begin{array}{l}\text { Finitura intermedia, giallo } \\
\text { scura }\end{array}$ & $* * * *$ & $*$ & $*$ & $\operatorname{tr}$ & * & * & ***** \\
\hline As3 & parte interna & $\operatorname{tr}$ & $* * *$ & $* * *$ & & $*$ & & $* * * * *$ \\
\hline As4 & $\begin{array}{l}\text { Finitura superficiale, di } \\
\text { colore giallo scuro }\end{array}$ & * $* * *$ & **** & $* * *$ & & $*$ & $* *$ & $* * * *$ \\
\hline As5 & Malta più interna & & ******* & ***** & & $*$ & $* *$ & $* * * *$ \\
\hline As6 & Malta scura & $*$ & $* * * *$ & $* * * *$ & & $*$ & $* *$ & $* * * *$ \\
\hline As7 & $\begin{array}{l}\text { Finitura di colore giallo } \\
\text { chiaro }\end{array}$ & $* * *$ & $* * * * *$ & $* * *$ & & $*$ & $* *$ & $* * * * *$ \\
\hline As8 & Finitura scura & $* * *$ & $* * *$ & $* * * * *$ & & $*$ & $* *$ & $* * * *$ \\
\hline As9 & $\begin{array}{l}\text { intonaco superficiale }+ \\
\text { finitura giallo chiaro }+ \text { fi- } \\
\text { nitura scura }\end{array}$ & & ******* & $* * *$ & & $*$ & $* *$ & $* * *$ \\
\hline As10 & $\begin{array}{l}\text { Deposito concrezionale } \\
\text { colore scuro, h } 20 \mathrm{~cm}\end{array}$ & & ****** & **** & $\operatorname{tr}$ & $*$ & $* *$ & $* * *$ \\
\hline
\end{tabular}

Tab. 1. Analisi in diffrazione ci raggi X (XRD).

\begin{tabular}{|c|c|c|c|c|c|c|c|c|c|}
\hline mil-eq & $\mathrm{Na}^{+}$ & $\mathrm{K}^{+}$ & $\mathrm{Mg}^{2+}$ & $\mathrm{Ca}^{2+}$ & $\mathrm{Cl}^{+}$ & $\mathrm{NO}_{3}$ & $\mathrm{PO}_{4}{ }^{*}$ & $\mathrm{SO}_{4}{ }^{=}$ & Oxal \\
\hline As1 & 9,87 & 3,88 & 9,85 & 44,7 & 6,66 & 21,8 & - & 23,6 & 0,70 \\
\hline As2 & 25,3 & 12,1 & 16,6 & 148,6 & 15,2 & 59,9 & - & 102,8 & 0,93 \\
\hline As3 & 15,1 & 7,25 & 12,3 & 37,2 & 10,1 & 38,6 & - & 12,2 & 0,17 \\
\hline As4 & 18,7 & 12,3 & 15,2 & 112,5 & 13,3 & 57,4 & - & 63,7 & 0,95 \\
\hline As5 & 9,46 & 6,75 & 11,7 & 29,9 & 7,98 & 29,3 & - & 5,54 & 0,22 \\
\hline AS6 & 9,68 & 5,88 & 7,84 & 37,9 & 7,90 & 28,4 & - & 16,4 & 0,35 \\
\hline AS7 & 0,90 & 0,92 & 5,27 & 78,2 & 0,81 & 2,15 & - & 60,9 & 0,87 \\
\hline AS8 & 2,91 & 4,67 & 4,78 & 54,3 & 3,04 & 20,7 & - & 31,5 & 0,43 \\
\hline AS9 & 1,84 & 2,56 & 5,03 & 20,2 & 1,51 & 12,8 & - & 3,77 & 0,21 \\
\hline AS10 & 6,13 & 3,62 & 12,0 & 75,2 & 5,46 & 81,1 & 0,62 & 2,68 & 1,34 \\
\hline
\end{tabular}

Tab. 2. Sali solubili espresso in milliequivalenti sulle malte.

I dati mostrano un'evidente omogeneità dei materiali presenti, come confermato dalle precedenti campagne diagnostiche. Questo ha aumentato la difficoltà nel riconoscimento, con queste tecniche analitiche, delle parti originali rispetto a quelle realizzate anche solo pochi anni dopo l'intervento di Giulio Romano, perché i materiali impiegati sono sostanzialmente gli stessi. Le analisi non invasive effettuate con aquaboy hanno mostrato come sulla superficie vi sia una 
grande capacità di assorbimento di acqua, cosa che è corrispondente alla presenza di gesso e naturalmente dovuta alla maggiore idrofilia del solfato rispetto al carbonato. I dati di XRF sono serviti per una verifica delle omogeneità superficiali e per caratterizzare le superfici colorate con relativi pigmenti. I "rossi", tema molto ripreso poi nella seconda fase di analisi, si presentano come "ocre", cosa che rende molto complicata la collocazione storica.

Le conclusioni emerse da questa prima fase precedente al cantiere pilota mettono in risalto l'uso di tinte a base di gesso in una data fase di intervento. Non si evidenziano evidenti fenomeni di risalita capillare, anzi l'umidità di superficie è in genere presente a partire dai $90 \mathrm{~cm}$ di altezza in su. La fase analitica di supporto al cantiere pilota ha tenuto conto di alcune problematiche legate anche alla formulazione delle malte da restauro e alla caratterizzazione di specifici materiali. Un tema particolare ha riguardato la colorazione rossa di alcune parti, quelle che le analisi XRF hanno definito composte da ocra rossa e non da cocciopesto come in prima ipotesi con evidenti ricadute ai fini della definizione esecutiva degli interventi. Nella tabella 3 è riportato l'elenco dei campionamenti con la tipologia di analisi prevista in funzione delle domande scaturite nel corso del cantiere pilota.

\begin{tabular}{|l|l|c|}
\hline & Descrizione del punto di prelievo & Analisi previste \\
\hline 1 & Finitura color violetto, metopa del fregio, Corridore & Stratigrafica \\
\hline 2 & $\begin{array}{l}\text { Finitura color giallo su violetto, triglifo del fregio, } \\
\text { Corridore }\end{array}$ & Stratigrafica \\
\hline 3 & Malta grigia della bugna, arco superiore, Corridore & Stratigrafica \\
\hline 4 & Malta gialla della bugna, arco superiore, Corridore & $\begin{array}{c}\text { Stratigrafica + } \\
\text { MR }\end{array}$ \\
\hline 5 & $\begin{array}{l}\text { Finitura rosa su grigio, tra una bugna e l'altra } \\
\text { dell'arco superiore, } \text { Corridore }\end{array}$ & $\begin{array}{c}\text { Stratigrafica + } \\
\text { MR }\end{array}$ \\
\hline 6 & $\begin{array}{l}\text { Scialbo grigio su finitura gialla, base della colonna } \\
\text { tortile, } \text { Rustica }\end{array}$ & $\begin{array}{c}\text { MO + SPOT } \\
\text { TEST + FTIR + } \\
\text { TGA }\end{array}$ \\
\hline 7 & $\begin{array}{l}\text { Finitura rosa su grigio, tra una bugna e l'altra, Cor- } \\
\text { ridore }\end{array}$ & $\begin{array}{c}\text { Stratigrafica + } \\
\text { MR }\end{array}$ \\
\hline 8 & $\begin{array}{l}\text { Malta grigia, base della seconda colonna tortile, } \text { Ru- } \\
\text { stica }\end{array}$ & $\begin{array}{c}\text { FTIR + XRD + } \\
\text { TGA + SPOT } \\
\text { TEST }\end{array}$ \\
\hline 9 & $\begin{array}{l}\text { Malta grigia con lacerti di scialbo rosato, base della } \\
\text { seconda colonna tortile, } \text { Rustica } \\
\text { dell'arco, } \text { Rustica }\end{array}$ & $\begin{array}{c}\text { Stratigrafica } \\
\text { MR }\end{array}$ \\
\hline
\end{tabular}

\begin{tabular}{|l|l|c|}
\hline 11 & $\begin{array}{l}\text { Boiacca dipinta, bugna a destra della lapide con } \\
\text { iscrizione, Mesi }\end{array}$ & $\begin{array}{c}\text { Stratigrafica + } \\
\text { MR }\end{array}$ \\
\hline 12 & $\begin{array}{l}\text { Materiale grigio sovrammesso, tra le bugne a sini- } \\
\text { stra della lapide, Mesi }\end{array}$ & $\begin{array}{c}\text { Stratigrafica + } \\
\text { MR }\end{array}$ \\
\hline 13 & $\begin{array}{l}\text { Finitura rosa-giallino, su malta sovrammessa } \\
\text { all'affresco, piano terra, Mesi }\end{array}$ & $\begin{array}{c}\text { Stratigrafica + } \\
\text { MR }\end{array}$ \\
\hline 14 & $\begin{array}{l}\text { Finitura rosa-cipria del fregio, quarto triglifo a parti- } \\
\text { re dall'angolo dx, Mostra }\end{array}$ & Stratigrafica \\
\hline 15 & $\begin{array}{l}\text { Finitura rosa-giallino della colonna tortile, angolo a } \\
\text { dx, Mostra }\end{array}$ & $\begin{array}{c}\text { Stratigrafica }+ \\
\text { MR }\end{array}$ \\
\hline
\end{tabular}

Tab. 3. Elenco punti di prelievo e analisi previste.

Da questi campioni si sono ottenuti risultati chiarificatori per quanto riguarda finiture presenti nel fronte del verso lago (Corridore). La sezione stratigrafica di un campione di rosso (Fig. 3) prelevato dal concio di chiave dell' arco mostra che non si tratta di una finitura colorata in pasta ma di una coloritura superficiale.

Diversa è la finitura riscontrata per le parti in bugnato grigie e gialle (Figg. 4 e 5) caratterizzate da un intonaco colorato in pasta con la superficie lavorata a bugnato.

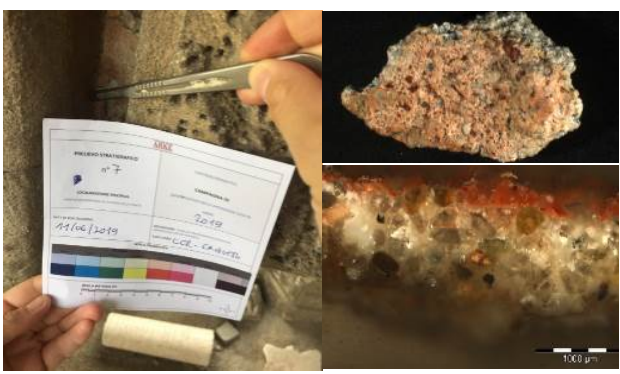

Fig. 3. Sequenza della lettura del campione numero 7, preso in parte nascosta e ipotizzata come originale dal restauratore: punto di prelievo fra i bugni e lettura del frammento superficiale e in sezione lucida.
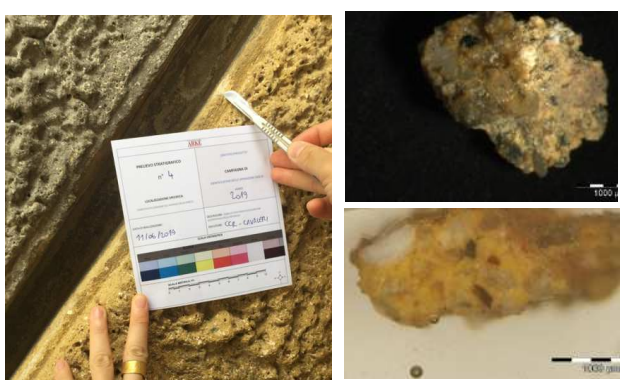

Fig. 4. Prelievo n. 4, parte colorata in giallo in pasta, e lettura del frammento superficiale e in sezione lucida. 
Lo studio ha riguardato anche la verifica di compatibilità delle malte da impiegarsi nel restauro con quelle originali, che risultano piuttosto omogenee pur nelle diverse fasi storiche. La base di valutazione è stata la misura dell'assorbimento dell' acqua mediante l'uso della tecnica delle spugnette per indirizzare le scelte verso l'impiego di malte con assorbimento simile rispetto a quelle originali; ciò per ridurre il rischio di migrazione di acqua, con il suo contenuto di sali, dalla malta nuova verso quella antica salvaguardo quella originale.

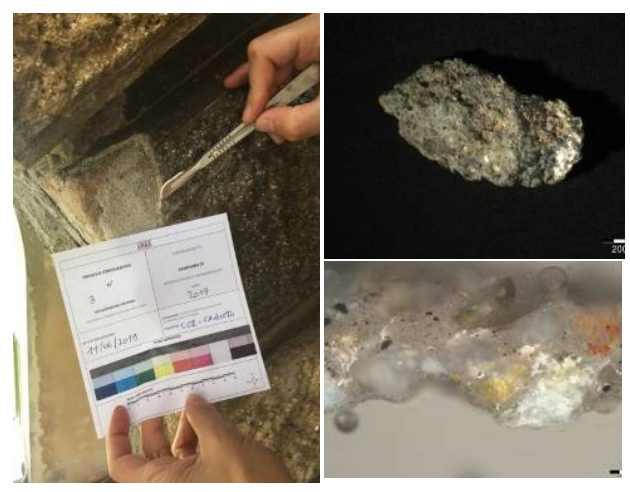

Fig. 5. Campione n. 3, parte colorata in pasta di colore grigio, lettura del frammento superficiale e in sezione lucida.

La morfologia articolata delle superfici non ha sempre permesso un confronto attendibile; inoltre molte malte antiche risultano lisciate in superficie, cosa difficile da replicare in caso di integrazioni di zone lacunose non troppo estese. Nella tabella 4 sono riportati i dati relativi a questa fase di sperimentazione.

\begin{tabular}{|l|l|c|c|c|c|}
\hline \multicolumn{5}{|c|}{ MISURE DI ASSORBIMENTO DELLE SUPERFICI ORIGINALI } \\
(TARGET) & $\mathrm{Pi}[\mathrm{g}]$ & $\mathrm{Pf}$ [g] & $\mathrm{Pi}$-Pf [g] & Wa \\
\hline A1 & $\begin{array}{l}\text { corridore, area bianco- } \\
\text { violaceo finto marmo a } \\
\text { sx del putto }\end{array}$ & 18,286 & 17,872 & 0,414 & $3,48 \%$ \\
\hline A2 & $\begin{array}{l}\text { corridore, area di malta } \\
\text { originale grigia }\end{array}$ & 18,705 & 18,013 & 0,692 & $5,82 \%$ \\
\hline A3 & $\begin{array}{l}\text { corridore, area di malta } \\
\text { originale gialla }\end{array}$ & 18,912 & 18,019 & 0,893 & $7,52 \%$ \\
\hline A4 & $\begin{array}{l}\text { rustica, area di malta } \\
\text { originale giallina }\end{array}$ & 18,666 & 18,169 & 0,497 & $4,18 \%$ \\
\hline A5 & $\begin{array}{l}\text { rustica, area di malta di } \\
\text { rifacimento }\end{array}$ & 18,758 & 17,889 & 0,869 & $7,31 \%$ \\
\hline
\end{tabular}

\begin{tabular}{|l|l|c|c|c|c|}
\hline A6 & $\begin{array}{l}\text { corridore, colonna torti- } \\
\text { le area bianca originale }\end{array}$ & 18,025 & 17,523 & 0,502 & $4,23 \%$ \\
\hline A7 & bugna grigia originale & 19,036 & 17,807 & 1,229 & $\begin{array}{c}10,35 \\
\%\end{array}$ \\
\hline \multicolumn{3}{|l|}{ MISURE DI ASSORBIMENTO DELLE SUPERFICI DI RESTAURO } \\
\hline $\begin{array}{l}\text { A1 } \\
\text {-r }\end{array}$ & $\begin{array}{l}\text { corridore, area restaura- } \\
\text { ta a sx del putto }\end{array}$ & 19,207 & 18,133 & 1,074 & $\begin{array}{c}9,04 \% \\
\text { A6 } \\
\text {-r }\end{array}$ \\
$\begin{array}{l}\text { lerridore, colonna torti- } \\
\text { area bianca di rifaci- } \\
\text { mento }\end{array}$ & 18,637 & 17,272 & 1,365 & $\begin{array}{c}11,49 \\
\%\end{array}$ \\
\hline $\begin{array}{l}\text { A7 } \\
\text {-r }\end{array}$ & $\begin{array}{l}\text { bugna grigia, area di ri- } \\
\text { facimento }\end{array}$ & 18,838 & 17,27 & 1,568 & $\begin{array}{c}13,20 \\
\%\end{array}$ \\
\hline
\end{tabular}

Tab. 4. Misure di assorbimento delle superfici originali, e di restauro.

\section{Il cantiere pilota}

Al fine di esemplificare la metodologia utilizzata nel cantiere pilota il contributo riporta i risultati riscontrati sul solo fronte della Rustica. Le indagini e le prove di intervento sono state eseguite su tutti i fronti.

La porzione della facciata esaminata nel corso del cantiere pilota ci ha mostrato un manufatto estremamente rimaneggiato nel corso dei secoli, poiché questo edificio è stato il primo ad essere edificato, con l'importante firma di Giulio Romano. Pertanto molte delle malte esaminate, si ritiene siano pressoché dovute ad interventi successivi. Interessante è stato notare come soltanto una porzione delle bugne del registro superiore, al confine con il Corridore, sono state realizzate con una malta grigia scura, colorata in pasta. Non sono stati effettuati prelievi stratigrafici su questa malta grigia, ma su di una porzione di malta simile, posta alla base della seconda colonna tortile: il risultato delle analisi sui prelievi n. 8 e 9, assimilabili in quanto effettuati sulla stessa porzione, a poca distanza l'uno dall'altro, ma sottoposti ad indagini differenti, descrive il materiale come un aggregato silicatico e legante carbonatico pigmentata da un nero organico. E' interessante notare la presenza di uno scialbo rosato, forse da attribuirsi ad un intervento di restauro che non apprezzava la presenza di cromie differenti ed ha uniformato le superfici scialbandole. Lo stato di conservazione di questa parte può essere considerato piuttosto buono. 
Si sono realizzati saggi stratigrafici per cercare di comprendere quali fossero le malte originali e verificate alcune delle operazioni da inserire nel progetto. Qui si riportano due esempi di campionatura effettuata.
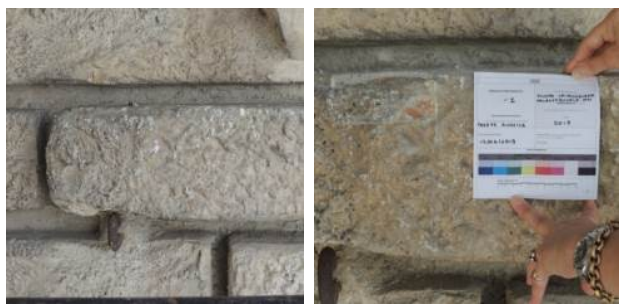

Fig. 6. Bugna rustica, a sinistra della finestra in alto, in parte in malta grigia, in parte in malta di calce e sabbia non pigmentata. 1.struttura muraria in laterizi che definiscono l'aggetto della bugna; non si riesce a leggere se tale struttura sia stata eseguita in due tempi; 2 . bugna suddivisa in due parti: i $20 \mathrm{~cm}$. di sinistra sono realizzati in malta lavorata, colorata di grigio nell'impasto; 3. malta della copertina, ovvero una stesura sulla porzione superiore delle malte, in calce e sabbia con inclusi di granulometria medio-fine; tale malta sembra non avere 4 . soluzione di continuità con quella della porzione di destra; tracce di scialbo a calce di color giallo pallido costituiscono la cromia residua.
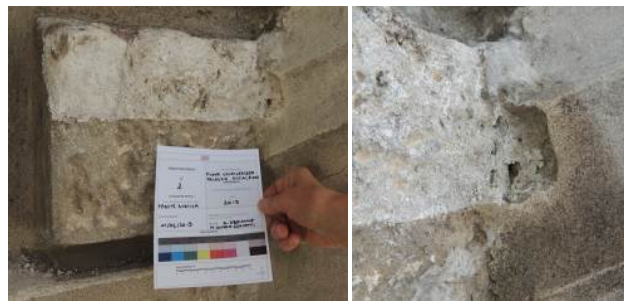

Fig. 7. Bugna a sinistra della colonna tortile e particolare della colonna tortile. 1. struttura muraria in laterizi che definiscono l'aggetto della bugna; 2 . arriccio in calce e sabbia di circa $5-6 \mathrm{~mm}$. di spessore; 3 . malta in calce e sabbia, lavorata; 4 . su di essa tracce di scialbo a calce di colore giallo pallido; 5 . sottile strato di malta in calce e sabbia, più grezza, 6 allargando il sondaggio su una piccola porzione della colonna, sotto una malta che si direbbe cementizia, di spessore variabile dai 2-3 ai 7-8 mm., vi è un sottile impasto bianco, molto liscio simile alla finitura degli elementi in stucco.

Dopo le indagini conoscitive sono state testate le possibili operazioni di intervento su ogni lato della Cavallerizza. Si riporta la sequenza delle prove eseguite sul fronte della Rustica e le osservazioni rispetto ad ognuna.

1. Spolveratura delle porzioni interessate dai sondaggi con pennelli morbidi;

2. Leggero risciacquo con acqua demineralizzata e tensioattivo a spruzzo, aiutandosi con spazzolini morbidi vista la scabrosità delle superfici. Il particellato atmosferico inquinante, più o meno adeso, si solubilizza con una discreta facilità senza apportare eccessiva umidità alle superfici. Sulle porzioni maggiormente ricarbonatate è stato necessario intervenire con impacchi localizzati di soluzioni leggermente basiche, avendo cura di interporre fogli di carta giapponese tra le superfici e la compressa, per facilitarne la rimozione;

3. Trattamento biocida con Biotin $\mathrm{T}$ al $3 \%$ in acqua demineralizzata, steso a pennello in due tempi. La prima volta è stato lasciato agire 5 giorni, la seconda 9 giorni. La superficie è stata poi risciacquata con acqua. L'operazione è stata eseguita nei saggi n. 7-9-10 (Fig. 8).

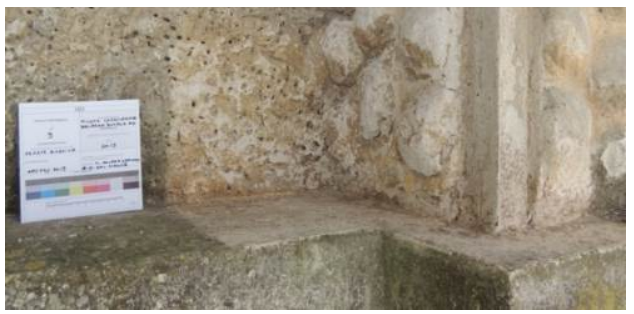

Fig. 8. Angolo tra la porzione laterale della base della colonna tortile e la bugna di fondo (saggio 9).

4. Consolidamento della malta microfratturata con resina acrilica in soluzione Acril 33 al 5\% in acqua demineralizzata, previa iniezione di acqua e alcool come veicolante. Si è testata la resina nanoacrilica, ma il suo comportamento non è stato altrettanto soddisfacente. Gli spessori ed i distacchi erano troppo esigui per una malta idraulica.

Si sono testate le operazioni anche sui pochi elementi lapidei naturali.

- Spolveratura e asportazione meccanica (a bisturi), di residui di guano. 
- Leggero risciacquo con spazzolini morbidi, acqua demineralizzata addizionata con poche gocce di tensioattivo (Desnovo);

- Impacco di carbonato d'ammonio in soluzione satura (180 gr/lt), addizionata con EDTA (sale bisodico dell'acido etlendiamminotetracetico) 30 gr/lt., utilizzando polpa di cellulosa e sepiolite in parti uguali come agente tixotropico, protetto con foglio di polietilene, viste le elevate temperature, lasciato agire per $8 \mathrm{~h}$.

- Risciacquo della superficie con acqua demineralizzata e spazzolini morbidi. Purtroppo, alcune piccole porzioni di particellato atmosferico inquinante (croste nere), non si sono solubilizzate. Si tratta di una porzione non visibile dal basso, per cui si è suggerito di scialbare a crema di calce e terre ventilate o nanocalci e terre ventilate (siena bruciata, terra di siena naturale, terra verde e nero di vite) piuttosto che utilizzare microsabbiature.

- Piccole stuccature, con una malta le cui cariche erano costituite da 2 parti di polvere di marmo Botticino 000, setacciato, 1 parte di polvere di marmo rosso Verona, $1 / 2$ parte di carbonato di calcio, $1 / 2$ parte di sabbia di fiume, come legante si sono usati calce idrata e calce idraulica Lafarge in rapporto 1:1. Il rapporto tra cariche e legante è di $3: 1^{1}$.

Dai cantieri pilota eseguiti su ogni fonte della Cavallerizza e sulle facciate è emersa una procedura operativa molto chiara. Con i dati ricavati, l'impostazione del progetto esecutivo risulta essere più sicura e con meno imprevisti, rischi di errori nell'interpretazioni degli effetti delle lavorazioni sui materiali, maggiore rispetto della materia ${ }^{2}$.

Il cantiere pilota costituisce una opportunità che nelle pieghe delle normative sugli appalti si configura come uno strumento guida operativo e amministrativo.

\section{Note}

${ }^{1}$ Tratto da: Lavori di restauro architettonico e delle superfici decorate di Corte Nuova, facciate della Rustica e della Cavallerizza verso il lago, presso il complesso museale di Palazzo Ducale a
Mantova. Relazione di restauro del cantiere pilota redatta da M. Chiara Ceriotti, Consorzio Arke'.

${ }^{2}$ Consolidamento laterizi decoesi: da due a più applicazioni a pennello e a siringa di silicato di Etile $\mathrm{OH}$ Wacker hanno dato un ottimo risultato. Consolidamento delle decoesioni: si ritengono migliori le Calosil piuttosto che le Nanorestore o le Nanorestore Plus, in quanto contengono una percentuale maggiore di nanocalci cui si aggiungono nanosilicati, per cui hanno una maggior capacità di penetrazione, non creano sbiancamenti superficiali e necessitano di un numero minore di applicazioni. Consolidamento di profondità: si è cercato di ridurre al minimo l'uso delle resine acriliche in emulsione (Acril 33), che pure ha dato risultati migliori rispetto alle resine nanoacriliche, mentre nel confronto tra il Ledan TB1 e il PLMA abbiamo riscontrato nel secondo caratteristiche più compatibili con il degrado presente, una migliore capacità di penetrazione grazie al superamento dello scoglio dato dalla polverosità all'interno dei distacchi. Pulitura: la parte superiore delle pareti e le porzioni più riparate, sono quelle su cui si riscontra la presenza maggiore di particellato atmosferico inquinante, più o meno ricarbonatato. Dopo l'indispensabile spolveratura con pennelli morbidi ed aspirapolvere a potenza graduabile, una leggera pulitura con acqua addizionata con poche gocce di tensioattivo può risolvere molte situazioni, ed è da considerarsi preliminare. Sulle croste nere o sulle ridipinture individuate, si suggerisce di ricorrere alla soluzione satura di carbonato d'Ammonio (180 gr/lt), addizionata o meno da un complessante (EDTA), in percentuale variabile (10-20-30-60 gr/lt), tenuta in sospensione in polpa di cellulosa e sepiolite, in modo da non cedere troppa umidità alla malta sottostante; se la superficie è scabra, si suggerisce di applicare la compressa su carta giapponese, in modo da facilitarne la rimozione. Trattamento biocida: abbiamo testato il Biotin $\mathrm{T}$ al $3 \%$ in acqua demineralizzata ottenendo ottimi risultati con $1 / 2$ applicazioni. Qualche difficoltà maggiore si è incontrata su malte particolarmente scabre poiché è problematica la rimozione dei biodeteriogeni all'interno dei fori o nelle asperità. Stuccatura: si sono testati tutti i tipi di malta sia colo- 
rate in pasta che solo lavorate, come si evince dalle ricette descritte sopra. L'unica malta di cui non si è riusciti a ritrovare la cromia è quella rossa, che si ritiene comunque troppo lacunosa e cromaticamente invasiva per essere riproposta, visti i risultati degli interventi precedenti. Per quanto riguarda le malte lavorate in calce e sabbia, si suggerisce di individuare una sabbia molto calda e di invecchiare poi i rifacimenti con velature a nanocalci leggermente pigmentate. $\mathrm{Si}$ suggerisce di utilizzare scialbi a nanocalci leggermente pigmentate, su tutte le porzioni molto estese di nuove stuccature per migliorarne l'aspetto e per renderle più compatte e resistenti agli agenti atmosferici. Si consiglia di intervenire in un primo tempo sulla facciata della Rustica e del Corridore, quindi su quella dei Mesi e della Mostra. Da non sottovalutare la situazione microclimatica che potrebbe interferire pesantemente su alcune delle operazioni previste.

\section{Bibliography}

Adami, A.; Della Torre, S.; Fregonese, L.; Mazzeri, A.; Scala, B.; Spezzoni, A. (2017). "Conoscenza e gestione del patrimonio costruito storico. Le nuove frontiere del BIM", in Atti del XXXIII Convegno di Studi Scienza e Beni Culturali, Arcadia Ricerche, Venezia, pp. 255-265.

Barbacci, A. (1939). "Il Cortile della Cavallerizza nel Palazzo Ducale di Mantova”, Palladio, III, 2, pp.63-76.

Bensi, P. (2016). "Alcune considerazioni sul controllo delle operazioni di restauro e sulla conservazione programmata dei dipinti murali”, in Della Torre, S., ed., Metodi e strumenti per la prevenzione e la manutenzione, Atti del convegno, Preventive and planned conservation: proceedings of the International Conference: Monza, Mantova, 5-9 May 2014, Nardini, Firenze, pp. 91-99.

Berzaghi, R. (1992). Il Palazzo Ducale di Mantova, Electa, Milano, p. 7.

Cottafavi, C. (1927). "Cronaca delle Belle Arti - R. Palazzo Ducale di Mantova. Appartamento dell'Estivale. Loggia dei Frutti”, in Bollettino d'Arte Ministero Beni Culturali e Ambientali (1926-1927), pp. 136-142, 421-428.

Cottafavi, C. (1934). "Palazzo Ducale di Mantova - La Galleria dei Marmi e la Loggia di Eleonora Medici”, in Bollettino d'Arte Ministero Beni Culturali e Ambientali (1933-1934), pp. 134-141.

Della Torre, S. (2003). La conservazione programmata del patrimonio storico architettonico, Linee guida per il piano di manutenzione e consuntivo scientifico, Guerini e Associati, Milano.

Della Torre, S. (2016). "La strategia della conservazione programmata: dalla progettazione delle attività alla valutazione degli impatti", in Della Torre, S., ed., Atti del convegno, Preventive and planned conservation: proceedings of the International Conference: Monza, Mantova, 5-9 May 2014, Nardini, Firenze.

L’Occaso S. (2002). Il Palazzo Ducale di Mantova, Electa, Milano, p 14.

Pagliara, P.N. (2002). "Materiali, tecniche e strutture in architetture del primo cinquecento", in Bruschi, A., Storia dell'architettura italiana. Il primo Cinquecento, Milano.

Paolucci, A. (1988). I Gonzaga e l'Antico: percorso di Palazzo Ducale a Mantova, edizioni Fratelli Palombi, Roma.

Togliani, C. (2003). "L’architettura da Fancelli a Giulio Romano", in Algeri, G., Il Palazzo Ducale di Mantova, Sonetti, Mantova, p. 114. 
